\title{
The First Results of the Low Voltage Cold-FE SEM/STEM System Equipped With EELS
}

\author{
Yu Yamazawa ${ }^{1}$, Satoshi Okada ${ }^{1}, Z^{2}$ lihuma Yasenjiang ${ }^{1}$, Takeshi Sunaoshi ${ }^{2}$ and Kazutoshi Kaji ${ }^{1}$ \\ ${ }^{1 .}$ Hitachi High-Technologies Corp., Electron Microscope Systems Design $1^{\text {st }}$ Dept., Ibaraki, Japan. \\ 2. Hitachi High-Technologies Corp., Application Development Dept., Ibaraki, Japan.
}

For various fields of research and development such as nano-materials, bio-materials or semiconductor devices, a role of electron microscope is becoming more important for not only image observation but also chemical characteristics analysis with high spatial resolution of nm or sub-nm level [1-3]. Since nano materials such as carbon nano-tube and graphene sheet are not strong against electrons accelerated to higher than $100 \mathrm{kV}$, it is one of effective approaches for reducing damages to lower accelerating voltage. Field-emission scanning electron microscope, Hitachi SU9000, performs to give not only secondary electron images including backscattered electron signals but also BF- and DF-STEM images at $30 \mathrm{kV}$ or lower voltage. BF-STEM lattice images at $30 \mathrm{kV}$ are detected for $\mathrm{Si}$ [222] lattice fringe, corresponding to $0.157 \mathrm{~nm}[4]$. In present study, we have developed the prototypes of an electron energy loss spectrometer and a diffraction camera unit for SU9000 in order to acquire not only element distribution and chemical information but also crystal structures at $30 \mathrm{kV}$ or lower voltage.

Figure 1 shows the configurations of SU9000 equipped with the EELS and the diffraction camera unit. Transmitted electron through specimen transfers into the EELS located under the column when the diffraction camera unit is retracted from the optical axis. The EELS system consists of two kinds of electron detectors; a spectrum detector with a two-dimensional CCD sensor $(256 \times 1024 \mathrm{ch})$ and an elemental distribution detector with a photomultiplier sensor consisting of 3 channels. The deflection coils after a dispersion coil serve as switching electrons to each detector. The minimum exposure time for spectrum measurement reaches to 1 micro second with the electric deflection function equipped in SU9000. A FWHM of a zero-loss peak is attainable to be better than $0.4 \mathrm{eV}$ at the acquisition time of 20 $\mathrm{ms}$ and $30 \mathrm{kV}$ as shown in Figure 2.

Figure 3 shows the fine structures of Ti-L edges in electron energy-loss spectra of $\mathrm{TiO}_{2}$ at $30 \mathrm{kV}$. The fine structures depending on the crystal structures of $\mathrm{TiO}_{2}$, rutile and anatase, clearly show the peak shift of about $1 \mathrm{eV}$ between two kinds of structures. SU9000 that performs the high spatial resolution image observation can provide the elemental and chemical information with high energy resolution by combing the prototype of EELS system

Figure 4 shows BF-STEM images of graphene sheet observed with different accelerating voltages of 30 and $200 \mathrm{kV}$, respectively. While continuously acquiring images in the same area at $200 \mathrm{kV}$, high resolution images and characteristic spots in the FFT images became more blurred due to the knockedon damage. But, in the case of $30 \mathrm{kV}$, even if observing in the same area for 10 times, no blurred images and spots were observed. Therefore, this results show that observation of graphene specimen at $30 \mathrm{kV}$ was useful to acquire the high resolution image with atomic level without damages.

References:

[1] K. Suenaga, M. koshino, Nature 468 (2010) 1088-1090. 
[2] U. Kaiser et al, Ultramicroscopy 111 (2011) 1239-1246.

[3] O. Kamimura et al, Ultramicroscopy 110 (2) (2010) 130-133.

[4] M. Konno, et al, Ultramicroscopy 145 (2014) 28-35.



Figure 1. Hitachi EELS system for SU9000



Figure 2. Zero Loss Peak of EEL spectra. (accelerating voltage $30 \mathrm{kV}$ )



Figure 3. Ti-L edges EEL spectra of $\mathrm{TiO}_{2}$ at $30 \mathrm{kV}$ (signal smoothing)


Figure 4. $1^{\text {st }}, 3^{\text {rd }}$ and $10^{\text {th }}$ scanning images of BFSTEM and FFT images of graphene at 30 and $200 \mathrm{kV}$. 